\title{
Survival Benefit of Transarterial Chemoembolization for Hepatocellular Carcinoma
}

\author{
Waqas Ahmad ${ }^{1}$, Iffat Rehman ${ }^{1}$, Osama Majeed ${ }^{2}$, Imran Khalid Niazi ${ }^{1}$, Khurram \\ Aftab Mufti ${ }^{3}$, Amjad Iqbal ${ }^{4}$
}

${ }^{1}$ Shaukat Khanum Memorial Cancer Hospital and Research Center Lahore, Pakistan. ${ }^{2}$ Imam Abdulrahaman Bin Faisal Hospital Dammam, Kingdom of Saudi Arabia. ${ }^{3}$ The Prince Charles Hospital Brisbane, Australia. ${ }^{4}$ Pakistan Kidney and Liver Institute Lahore, Pakistan.

\begin{abstract}
Objective: Transarterial chemo-embolization (TACE) is a palliative treatment option for hepatocellular carcinoma (HCC) with improved patient survival. The aim of the study was to see the outcome of our patients at our institution 2 years post TACE. Patients and Methods: Electronic records were retrospectively reviewed for patients who had TACE from $1^{\text {st }}$ November $2009-31^{\text {st }}$ October 2012. Baseline imaging, multidisciplinary team (MDT) and clinical notes, pathology labs, TACE angiograms and follow up imaging were reviewed for 2 years after first TACE. Procedure complications, clinical status and findings at follow up CT were reviewed and analyzed in SPSS version 19. Survival was assessed using Kaplan Meier curves. Results: A total of 104 patients had TACE for HCC. Amongst these 21 patients were lost to follow up and could not be contacted to reconfirm the outcome and had to be excluded to minimize bias. Amongst the included 83 patients, $57(68.7 \%)$ were male and $65(78.4 \%)$ were 51-70 years of age at time of first TACE. The commonest cause for HCC was $\mathrm{HCV}$ in $74(89.2 \%)$ patients. Target lesion size at baseline CT was $<5 \mathrm{~cm}$ in $38(45.8 \%)$ patients, $5-10 \mathrm{~cm}$ in 37 (44.6\%) patients and $>10 \mathrm{~cm}$ in $8(9.6 \%)$ patients. A total of $25(30 \%)$ patients needed more than 1 session of TACE. On post TACE CT, 46 (55.4\%) patients had good packing of lipoidol in the lesion. A total of $18(21.7 \%)$ patients progressed in TACED lesions while another $21(25 \%)$ progressed with new lesions. One patient had metastasis to adrenal gland. Only $8(9.6 \%)$ patients had liver failure after TACE and 1 patient had hepatorenal syndrome. Only 1 patient died within 30 days after TACE. Two years post TACE, 47 (56.6\%) patients were alive indicating good outcome. Conclusion: TACE improves survival in HCC; 1 year survival was $80 \%$ and 2 year survival was $56.6 \%$.
\end{abstract}

Keywords: Heptocellular carcinoma-Transarterial chemoembolization- survival- outcomes- Oncology

\section{Introduction}

HCC is the fifth most frequent cancer in the world and the third most common cause of cancer-related mortality in the world [1-2]. The incidence of hepatocellular carcinoma is rising worldwide due to the $\mathrm{HBV}$ and $\mathrm{HCV}$ epidemics and the successful treatment of other complications of cirrhosis [3]. The diagnosis is made based on imaging features on multiphasic abdominal CT scan or dynamic MRI imaging and correlating it with serum alpha-feto protein levels. Alpa-feto protein is the only serological marker commonly used in diagnosis, but has a poor sensitivity ranging from $39 \%$ to $65 \%$ and a specificity ranging from $76 \%$ to $97 \%$ [4]. In case of atypical imaging features, the lesion is subjected to biopsy. Decision on biopsy of a focal liver lesion should be discussed by the multidisciplinary team, including a hepatobiliary and transplant surgeon. There is a risk of tumor seeding during biopsy varies between $0 \%$ and $11 \%$. [5]. Despite the widespread use of screening programs, $60-70 \%$ of $\mathrm{HCCs}$ are detected when curative treatments (surgery or ablation) are precluded by the cancer burden or

Corresponding Author:

Dr. Waqas Ahmad

Department of Radiology Shaukat Khanum Memorial Cancer Hospital and Research Center, Lahore, Pakistan.

Email:waqasrad@gmail.com 
contraindications [6].

Among the available curative and palliative treatment options, Transcatheter arterial chemoembolisation (TACE) is the most common and treatment of choice for intermediate stage hepatocellular carcinoma (HCC) [2][7].

TACE includes the selective injection through the hepatic artery of antineoplastic agents (e.g. cisplatin, doxorubicin, mitomycin), together with selective obstruction of tumoral feeding vessels (e.g. with coils, gelatine sponge particles). TACE yields response rates of $35-42 \%$ and prolongs life in comparison with best supportive care in selected patients with intermediate advanced HCC (Child-Pugh A, no portal invasion or extrahepatic metastases) [9].

The rationale of TACE is unique dual hepatic blood supply. In contrast to normal and regenerating liver in cirrhosis which gets nearly $80 \%$ blood supply from portal vein, HCC receives its blood supply predominantly through the hepatic arterial system. Therefore TAE or TACE has a marked anti-tumoral effect while only minimally affecting the surrounding liver parenchyma.

\section{Materials and Methods}

\section{Patient Selection}

The retrospective study was conducted in which the electronic records of patients who had TACE from $1^{\text {st }}$ Nov 2009 to $31^{\text {st }}$ Oct 2012 were reviewed. Each patient was selected for TACE after discussion in MDT. Baseline imaging, multidisciplinary team (MDT) and clinical notes, pathology labs, TACE angiograms and follow up imaging were reviewed for 2 years after first TACE. Procedure complications, clinical status and findings at follow up CT were reviewed. Patients who had lost to follow up at 2 years or their family was contacted on phone and inquired about current status. Non-responders were excluded.

\section{Procedure details}

Patients underwent TACE from the common femoral artery approach after informed written consent and review of fresh labs. Celiac and superior mesenteric angiograms were obtained to assess arterial anatomy and tumor vasculature. The chemotherapeutic agent (doxorubicin) mixed with lipiodol was than infused into the feeding artery and stasis obtained.

\section{Follow-up}

Follow up imaging was performed. Data of clinical notes, pathology labs for monitoring of ascites, hepatic encephalopathy and liver function tests was also reviewed.

\section{Imaging protocol}

Pre-treatment CT or MRI scans were used for documenting the initial findings. The radiological requirement required for discussion in the $\mathrm{HCC}$ conference was either a biphasic contrast-enhanced CT or dynamic MRI. Local response to therapy was documented 6 weeks after TACE using triphasic CT scan acquiring unenhanced, arterial and portal venous phases. The response to therapy was reassessed by review of imaging by four experienced radiologists having experience of more than 5 years. The basis for this was comparison of the initial finding with the last documented examination of the patient.

\section{Objective}

The objective of this study was to assess the overall patient outcome at our Hospital and compare with international standards.

\section{Statistical Analysis}

All the collected data entered into SPSS version 17 and analysed. Data was stratified according to age, gender, child Pugh score [8], aetiology, lesion size at baseline, uni or multifocality of lesions, involvement of single or both lobes, selective/non-selective TACE, hepatic artery embolization, number of sessions and lipiodol packing on post-TACE scan. Kaplan-Meir curve was used to estimate survival up to two years (Figure 1).

\section{Results}

A total of 104 patients had TACE for HCC. Amongst these 21 patients were lost to follow up and even their families could not be contacted to reconfirm the outcome and had to be excluded to minimize bias. Amongst the included 83 patients, $57(68.6 \%)$ were male and $26(31.33 \%)$ were female. Age of 65 patients $(78.4 \%)$ ranged between 51-70 years at time of first TACE with the mean age of overall study population to be 59.94 years. The commonest cause for HCC was HCV in 74 (89.2\%) patients. 78 patients $(94 \%)$ had Child's score A while 5 patients $(6 \%)$ had child's score B [8]. Target lesion size at baseline CT was $<5 \mathrm{~cm}$ in $38(45.8 \%)$ patients, $5-10 \mathrm{~cm}$ in $37(44.6 \%)$ patients and $>10 \mathrm{~cm}$ in $8(9.6 \%)$ patients. In terms of disease focality; 34 patients $(40.96 \%)$ had bifocal disease, 30 patients $(36.14 \%)$ had unifocal disease while $19(22.89 \%)$ had multifocal disease. 59 patients (71.1\%) had unilobar disease while both hepatic lobes were involved in 24 patients $(28.92 \%) .77$ patients $(92.3 \%)$ underwent non-selective TACE. Three patients had selective TACE while 3 patients had more than one session which included selective as well as non-selective TACE. Hepatic artery was embolized in 77 patients (92.3\%). 58 patients $(69.9 \%)$ underwent only one session of TACE while 20 patients $(24.1 \%)$ had two sessions.5 patients $(6.01 \%)$ underwent three sessions of TACE.

\section{Lipiodol packing on Post-TACE CT}

Patients having over $75 \%$ of the lesion packed with lipiodol were labelled to have good packing, between $50-75 \%$ were labelled to have mostly packed while those with less than $50 \%$ of packing were labelled to have patchy packing (Figure 2).

\section{Tumor response}

Tumor response was classified under the headings of completed response, partial response, stable disease, progression in lesion subjected to TACE, progression with development of new lesion and progression with metastatic disease (Figure 3). 


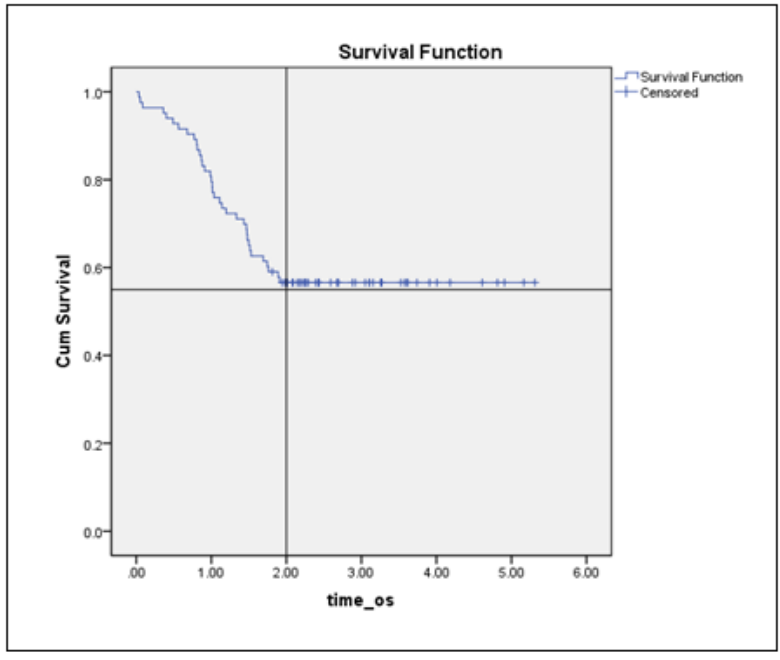

Figure 1. Kaplan Meier Curve Indicating 1 Year Survival of $80 \%$ and 2 Year Survival of $56.6 \%$ Post TACE

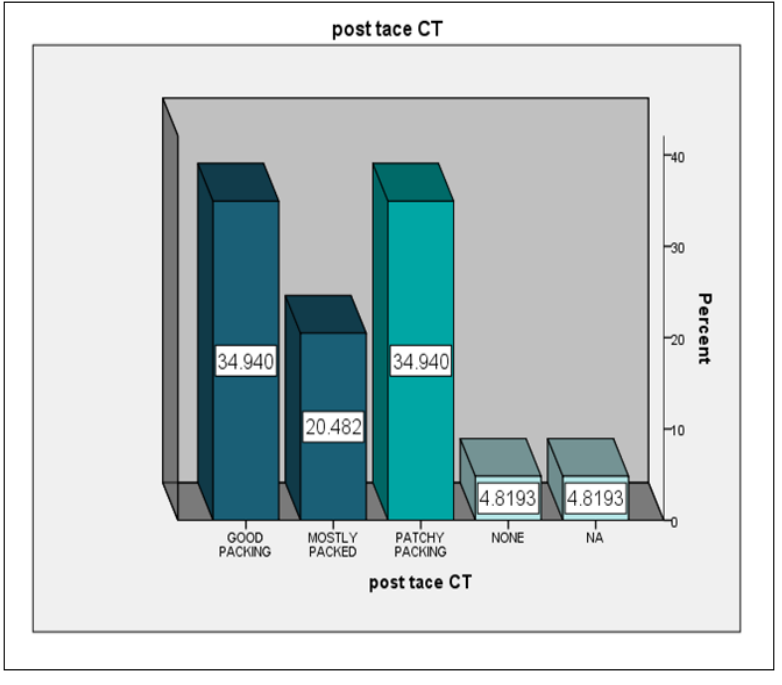

Figure 2. Lipiodol Packing Percentage

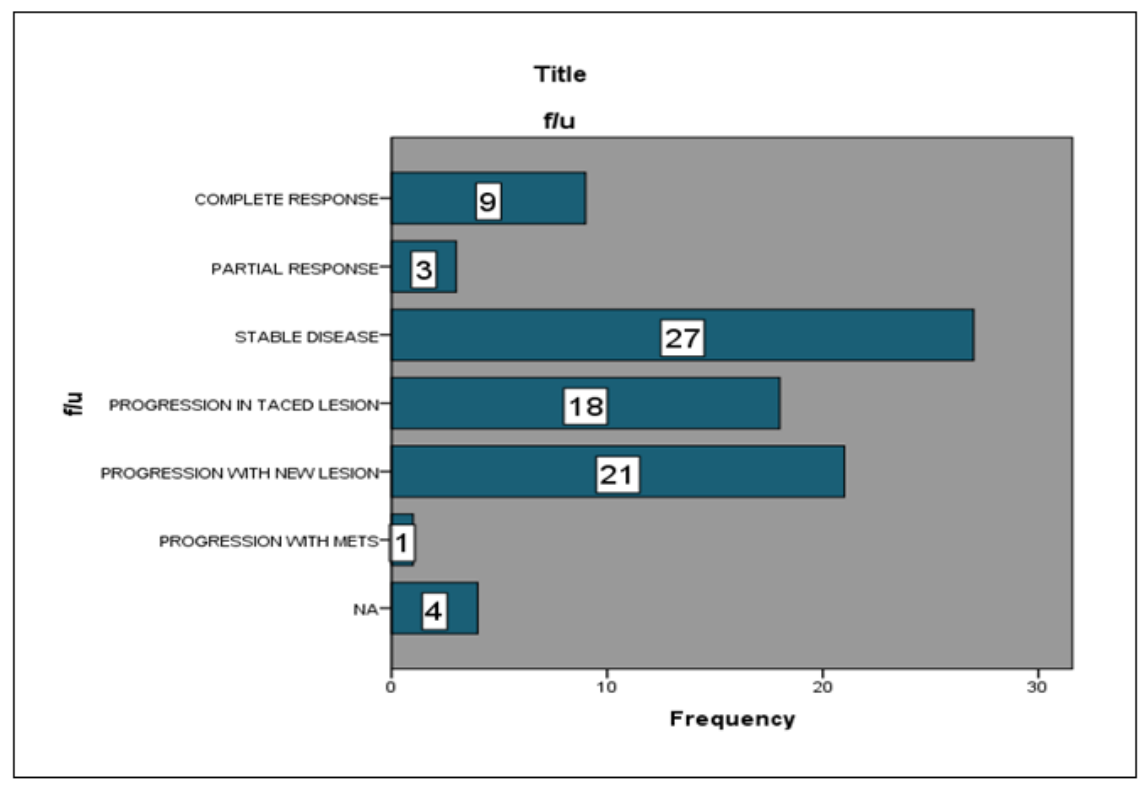

Figure 3. Response Assessment of TACE

\section{Complications}

Complications were classified under the headings of chemo related side effects, hepato-renal syndrome, Liver failure and death (Figure 4).

\section{Survival rate}

Our study indicated 1 year survival of $80 \%$ and 2 year survival of $56.6 \%$ post TACE (Figure 5).

\section{Discussion}

Golfieri et al [2] published a study in 2014 comparing two year survival of conventional TACE (cTACE) versus DEB-TACE [2]. The estimated two year survival of cTACE was $55.4 \%$ as compared to $56.6 \%$ of our study. Their study population included 88 patients. $13.5 \%$ patients were Child's B as compared to our $6.02 \%$. Additionally, the chemotherapeutic agent used

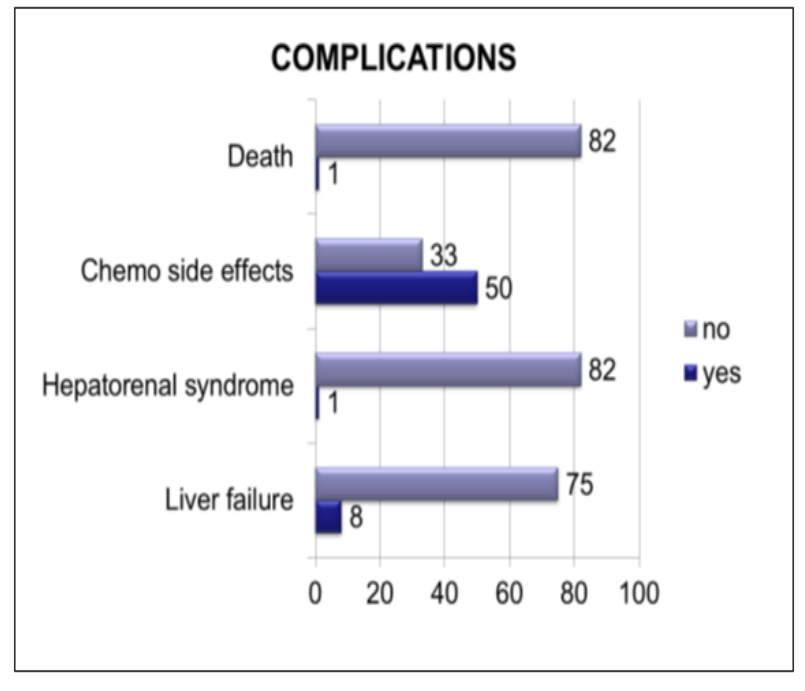

Figure 4. Complication in Percentages 


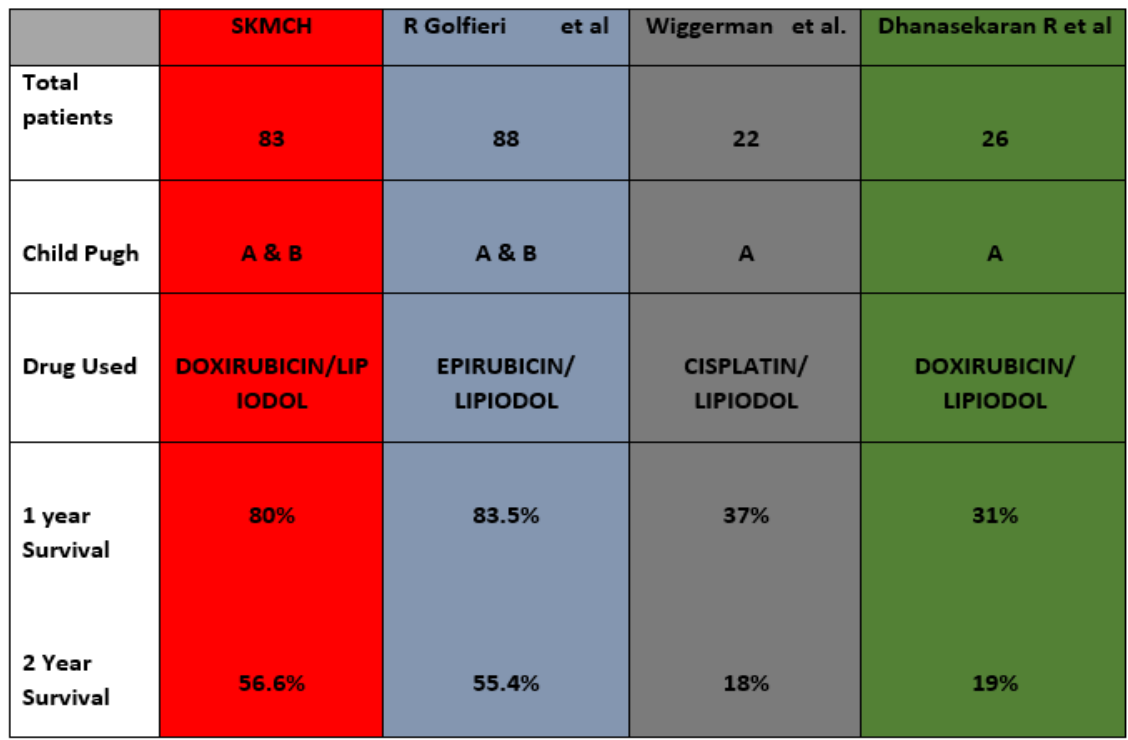

Figure 5. Comparative Analysis of Different Studies

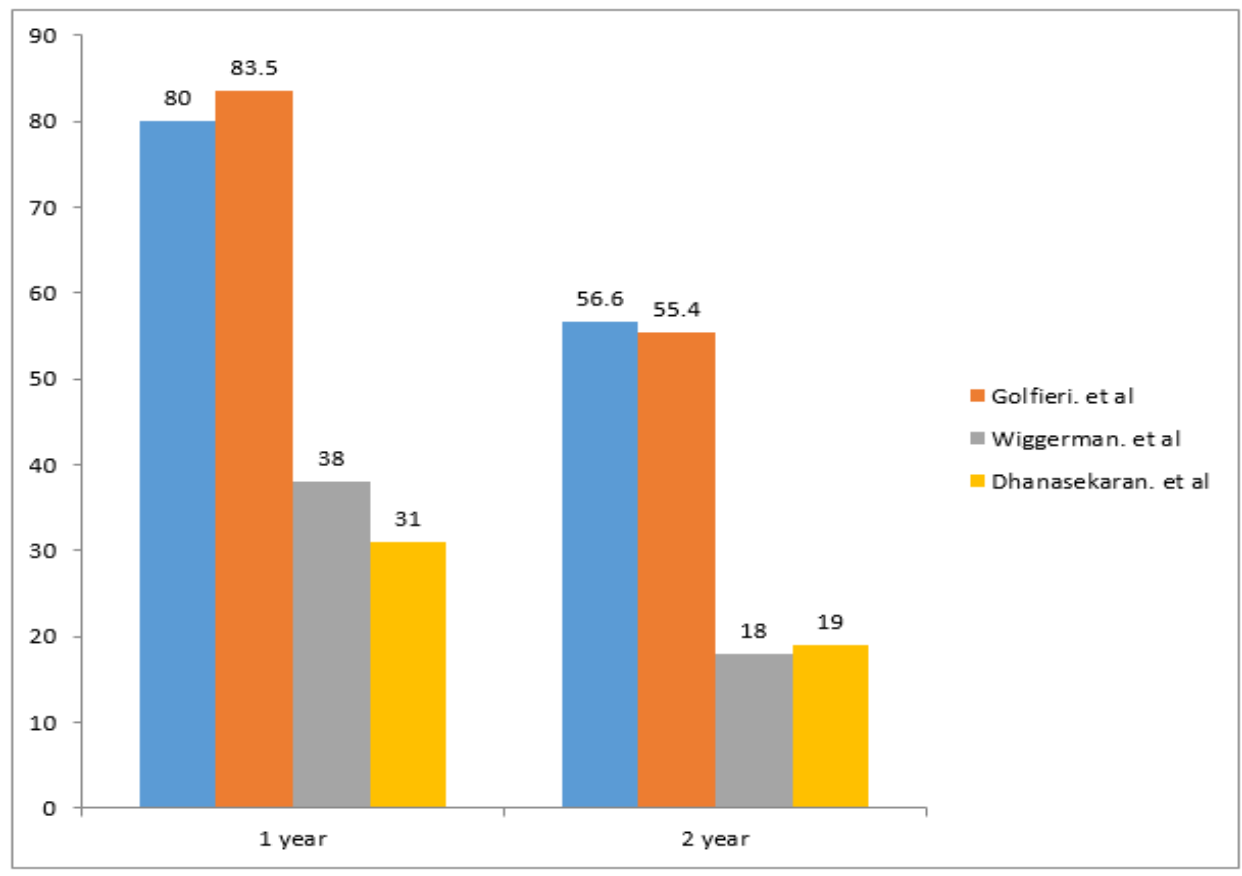

Figure 6. 1 and 2 Year Survival Rates of Different Studies

was epirubicin. Another study conducted by Dhanasekaran R et al [10] from January 1998 up to July 2008 studying the comparing the outcome of cTACE with DEB-TACE showed a two year survival of $19 \%$ for cTACE [10]. 26 out of the total of 71 of their patients underwent cTACE. $100 \%$ of their patient undergoing cTACE were child's A. Cisplatin was the chemotherapeutic agent used in their study population. Wiggerman et al. [11] conducted a study in patients undergoing TACE from 2003-2008 which included 74 patients out of which 22 underwent cTACE [11]. All the 22 patients were child's A, receiving cisplatin as chemotherapeutic agent. The estimated 2 year survival rate was $18 \%$ (Figure 6).

\section{Limitations}

1.21 patients were excluded from the total population who lost to follow up.

2. Comparative studies compared cTACE with the DEB-TACE while our studies included the patients undergoing cTACE.

3. Child's score

4. Drug used 


\section{References}

1. Kamangar F, Dores GM, Anderson WF. Patterns of Cancer Incidence, Mortality, and Prevalence Across Five Continents: Defining Priorities to Reduce Cancer Disparities in Different Geographic Regions of the World. Journal of Clinical Oncology. 200605 10;24(14):2137-2150. https://doi. org/10.1200/jco.2005.05.2308.

2. Golfieri R, Giampalma E, Renzulli M, Cioni R, Bargellini I, Bartolozzi C, Breatta AD, Gandini G, Nani R, Gasparini D, Cucchetti A, Bolondi L, Trevisani F. Randomised controlled trial of doxorubicin-eluting beads vs conventional chemoembolisation for hepatocellular carcinoma. British Journal of Cancer. 201406 17;111(2):255-264. https://doi. org/10.1038/bjc.2014.199.

3. Chang M, Chen C, Lai M, Hsu H, Wu T, Kong M, Liang D, Shau W, Chen D. Universal Hepatitis B Vaccination in Taiwan and the Incidence of Hepatocellular Carcinoma in Children. New England Journal of Medicine. 199706 26;336(26):18551859. https://doi.org/10.1056/nejm199706263362602.

4. Giannelli G, Antonaci S. New frontiers in biomarkers for hepatocellular carcinoma. Digestive and Liver Disease. 2006 Nov;38(11):854-859. https://doi.org/10.1016/j. dld.2006.05.007

5. Stigliano R, Marelli L, Yu D, Davies N, Patch D, Burroughs A. Seeding following percutaneous diagnostic and therapeutic approaches for hepatocellular carcinoma. What is the risk and the outcome?. Cancer Treatment Reviews. 2007 08;33(5):437-447. https://doi.org/10.1016/j.ctrv.2007.04.001

6. Takayasu K, Arii S, Kudo M, Ichida T, Matsui O, Izumi N, Matsuyama Y, Sakamoto M, Nakashima O, Ku Y, Kokudo N, Makuuchi M. Superselective transarterial chemoembolization for hepatocellular carcinoma. Validation of treatment algorithm proposed by Japanese guidelines. Journal of Hepatology. 2012 04;56(4):886-892. https://doi. org/10.1016/j.jhep.2011.10.021.

7. Varela M, Real MI, Burrel M, Forner A, Sala M, Brunet M, Ayuso C, Castells L, Montañá X, Llovet JM, Bruix J. Chemoembolization of hepatocellular carcinoma with drug eluting beads: Efficacy and doxorubicin pharmacokinetics. Journal of Hepatology. 2007 03;46(3):474-481. https://doi. org/10.1016/j.jhep.2006.10.020.

8. Verslype C, Van Cutsem E, Dicato M, Arber N, Berlin J, Cunningham D, De Gramont A, Diaz-Rubio E, Ducreux M, Gruenberger T, Haller D, Haustermans K, Hoff P, Kerr D, Labianca R, Moore M, Nordlinger B, Ohtsu A, Rougier P, Scheithauer W, Schmoll H, Sobrero A, Tabernero J, van de Velde C. The management of hepatocellular carcinoma. Current expert opinion and recommendations derived from the 10th World Congress on Gastrointestinal Cancer, Barcelona, 2008. Annals of Oncology. 2009 06;20:vii1-vii6. https://doi.org/10.1093/annonc/mdp281.

9. Llovet JM, Real MI, Montaña X, Planas R, Coll S, Aponte J, Ayuso C, Sala M, Muchart J, Solà R, Rodés J, Bruix J. Arterial embolisation or chemoembolisation versus symptomatic treatment in patients with unresectable hepatocellular carcinoma: a randomised controlled trial. The Lancet. 2002 05;359(9319):1734-1739. https://doi. org/10.1016/s0140-6736(02)08649-x.

10. Dhanasekaran R, Kooby DA, Staley CA, Kauh JS, Khanna V, Kim HS. Comparison of conventional transarterial chemoembolization (TACE) and chemoembolization with doxorubicin drug eluting beads (DEB) for unresectable hepatocelluar carcinoma (HCC). Journal of Surgical Oncology. 2010;:n/a-n/a. https://doi.org/10.1002/jso.21522

11. Wiggermann P, Sieron D, Brosche C, Brauer T, Scheer F,
Platzek I, Wawrzynek W, Stroszczynski C. Transarterial Chemoembolization of Child-A hepatocellular carcinoma: Drug-eluting bead TACE (DEB TACE) vs. TACE with Cisplatin/Lipiodol (cTACE). Medical Science Monitor. 2011;17(4):CR189-CR195. https://doi.org/10.12659/ msm. 881714

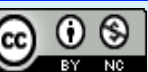

This work is licensed under a Creative Commons AttributionNon Commercial 4.0 International License. 